\title{
Automation approach for Unity based multi-player card game
}

\author{
Sajid Shaik, Pragna P. Pal \\ Junglee Games India Private Limited, \\ Bengaluru- 560034, India \\ shaiksajid.in@gmail.com,pragnapal29@gmail.com
}

Abstract-- When it comes to Unity-based[3] applications, there are very few tools in the market that may be viable as an option to test out journey-based games with components that are predictable in the game but what about unpredictable games like solitaire, rummy? There are tools that make components of the Unity application visible which can be validated using the unique ids but this isn't the case for a game with only cards and especially when we don't know the state of the game and the deck layout. This paper discusses the approach to bring efficient, generic, and reusable solutions for challenges while automating the logic validation of a card game layout for each move done. The automation approach spans the following areas: 1) The mechanism for detecting the native elements 2) Approach for customizing the element names and ids uniquely 3) Automating the game as per the standard logic by creating separate functions that take native objects as parameters.

The benefit of this automation approach is that it runs on any device/emulator irrespective of the varying screen size and resolutions and across whatever the deck layout maybe. The client is done on python with a Unity platform support tool "AirtestIDE"[1] developed by NetEase[2] and the element detection is done through Poco[4] manager. We customized the element ids for each card with precision considering the attributes that will be required to carry out the automation flow.

\section{INTRODUCTION}

An application that has predictable user-based flows available on the UI is easy to automate, unlike solitaire that requires extensive use of card logics and sequences to test out the game move scenarios. The automation done for a card-based game developed on a Unity platform is not only difficult to automate in terms of the platform used but also for understanding the underlying approach for getting the logic right for move validation in the game. There is no known prediction for the game deck at any point in time. Therefore, this may boil down to the question of how is the validation carried out? To overcome this major roadblock, the proposed methodologies were 1)to customize the elements that were visible in the IDE as per the card suit, rank, and the card face visibility 2) to divide the game board into different regions where the cards are bundled/arranged 3)to create small utilities per se modules that are called to get the information related to the card/card bundle as per the customized element details provided during game development, this approach makes it easier to handle the unpredictable behaviour of the game. This approach was developed with a vision to have a unified automation code that could be used to run functional test suites for validating client and server logic of any gameplay. 


\section{LITERATURE SURVEY}

The major building block that drove the logical validation of the game was element recognition and customization which covered the basic

- We need to recognize the elements i.e. the cards and the card region so that we can just get the information of the movement of the cards from the source to the destination to validate the game logic.

- The application is built using the naming conventions containing the card-related information.

- Each card objects are categorized as per the uniqueness of each card in the card deck with each name containing the card suit (Diamonds, Clubs, Hearts, Spades), card rank (A,2,3,4,5,6,7,8,9, J, Q, K), card colour (Red, Black). Regions for card locations are defined and dynamically the regions' objects are assigned to the cards once card movement happens.

- Movements of the cards validated if the logical placing is correct or not with defined expected results and the actual results after movement.

- To ensure the sync of the server and client movements, the server response after each successful client movement is tracked down and passed to the IDE through an interface. The client and the server movements are validated if they are in sync.

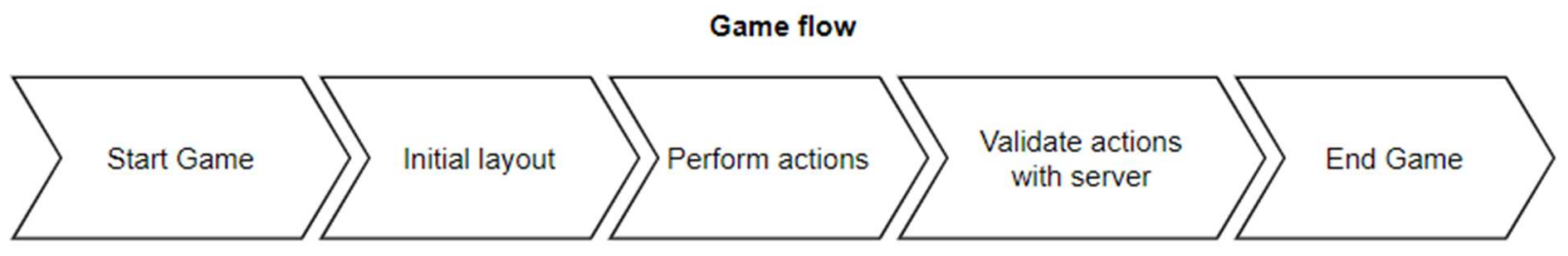

Fig. 1: Order of the game flow

An example of sample data:

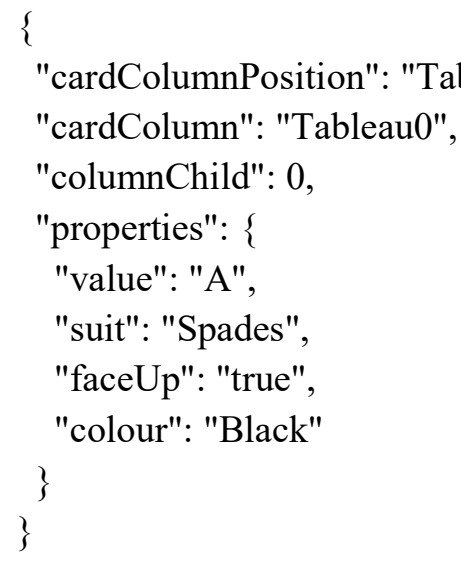

$\rightarrow$ The column within the region

$\rightarrow$ The node within the column where card lies

$\rightarrow$ The rank of the card from A to K (13 values)

$\rightarrow$ The suits (Diamonds, Clubs, Hearts, Spades)

$\rightarrow$ The card face visibility to user

$\rightarrow$ The colours (Red, Black) 
Below are some tools based on the element object-based recognition, discussed for Unity application. 1. AirtestIDE:

- AirtestIDE is an open-source testing tool by NetEase which is useful for detecting elements in a Unity-based application. This makes use of the Poco manager to detect the element objects and also for the actions performed on the application.

- It has an inbuilt ADB connection which allows any device to be easily connected for running the application under test. It also has a remote connection to connect to cloud/non-cloud port for emulators

- It supports various platforms and for our use case it has a Poco assistant that helps in getting the object reference of the objects on the screen for Unity based applications

- It supports only python client for scripting

2. Object-based Recognition:

Object-based Recognition involves capturing the properties or attributes of the visible elements in the current frame like visible text, unique identifier, etc., Object-based Recognition allows using more than a single attribute for identifying the element uniquely, like using XPath and thus making less error-prone in locating the visible element in the currently visible frame.

Limitations:

- Mechanism to read the properties or attributes of the visible element in the current frame is required.

\section{SOLUTION BY JUNGLEEGAMES:}

The approach for automation is to create a communication channel between the Unity platform and the Airtest. The approach for automation is to provide the objects library. We achieved this by utilizing an open-source SDK manager called "Poco". This library lets us get access to the elements object references, and properties. Once this is integrated into the game project then the initialization of the Poco manager happens during the game launch.

The device is connected to the Airtest IDE via ADB connection and we are ready to create a new project on the IDE. The game properties are accessed from the game with the help of the hierarchy manager in the Airtest ide.

The approach for automation is to provide the object of the source and the destination when a card movement happens. The elements are recognized by the Poco manager and the customized attribute values are available for testing. The Poco manager returns all the data properties as per the visible elements in the current frame. We access certain properties of the elements to define the navigation from one point to another as per our requirement. "The manipulation of the size" and the orientation of elements also can be done with the predefined Poco libraries.

There were some cons w.r.t the Airtest ide:

1. All the properties of an element are inaccessible. We overcame this by supplying all the data for the particular element into one of the data attributes of the element which we made accessible using Poco object. 
2. The hierarchical order of the current frame wasn't getting refreshed until the manual trigger for refreshing on the IDE.

3. The communication was only one-sided which is from Unity to Airtest but not the other way around. This could have opened up a world to new possibilities.

There are situations where we want to access customized data from the game on the Airtest IDE and that can be achieved by using Poco manager of the Unity to let the Airtest IDE access those customized data created on the game.

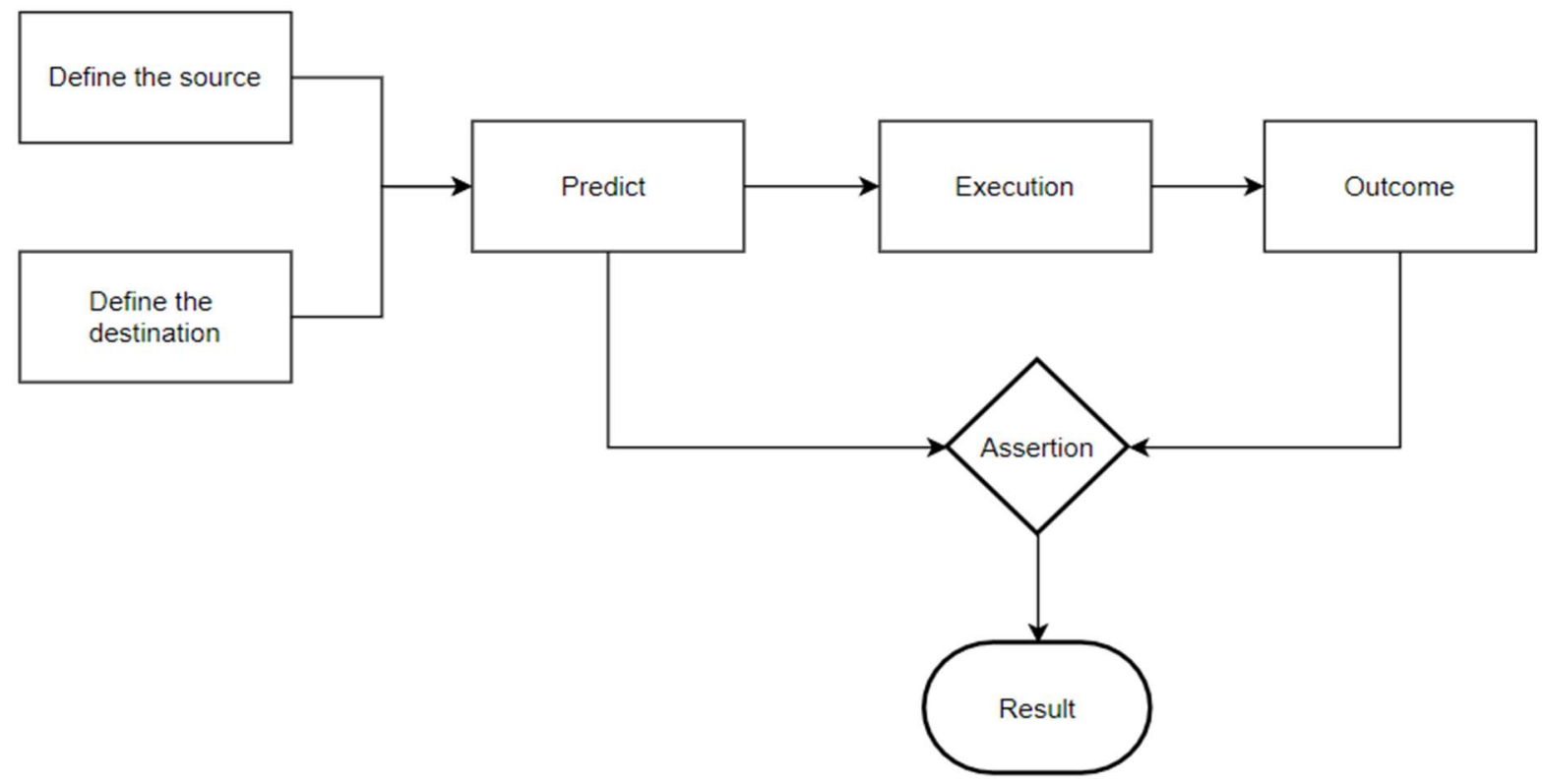

Fig. 2: Automation flow structure

\section{PROPOSED SYSTEM}

We use Solitaire as an example to demonstrate the approach but this can also be used for any card-based games by changing The proposed solution contains the following layers:

Front-end Layer

1. The Unity layer contains the build properties and configuration related to the game board and card movements. The client-level logics are defined on this platform which creates the backbone of the game.

2. The Poco layer is responsible for providing the inlet of communication between the two tech stacks. Poco provides accessibility to the game objects to get the object properties. The customized game objects which are defined in the Unity game layer are since made accessible to the Poco manager in the Airtest ide, this opens up the scope for automating the game logic.

3. Generic methods are created for getting the card-related data, the card count in a region, and the list of cards contained in a region and consecutively do the movement from source to destination. The use of Poco manager here is to get the region and card elements and respectively return the 
desired data. The validation as per the use cases is done on the game board for card positions and movements by utilizing the pre-created generic methods.

4. The gameplay logic helps in the validation of the test cases related to card navigation. With this, the expected result of the card movement is stored in a memory. The execution of the test case happens in its card movement action. Thereafter the actual result is checked following by the comparison of the expected and actual results. The client-server logic validation is attained.

\section{Backend Layer}

In our specific use case, we have set up the server in such a way that it validates every move from the client end to avoid tampering on the client. We make use of this specific setup to validate our test cases by generating fake/real moves and sending them to the server and we get authenticity back in response. This has been done by use of a separate manager entity called Socket manager which exposes the response that was received from the server. This avoids any heavy computations on the client-side thus, making the test cases execution faster.

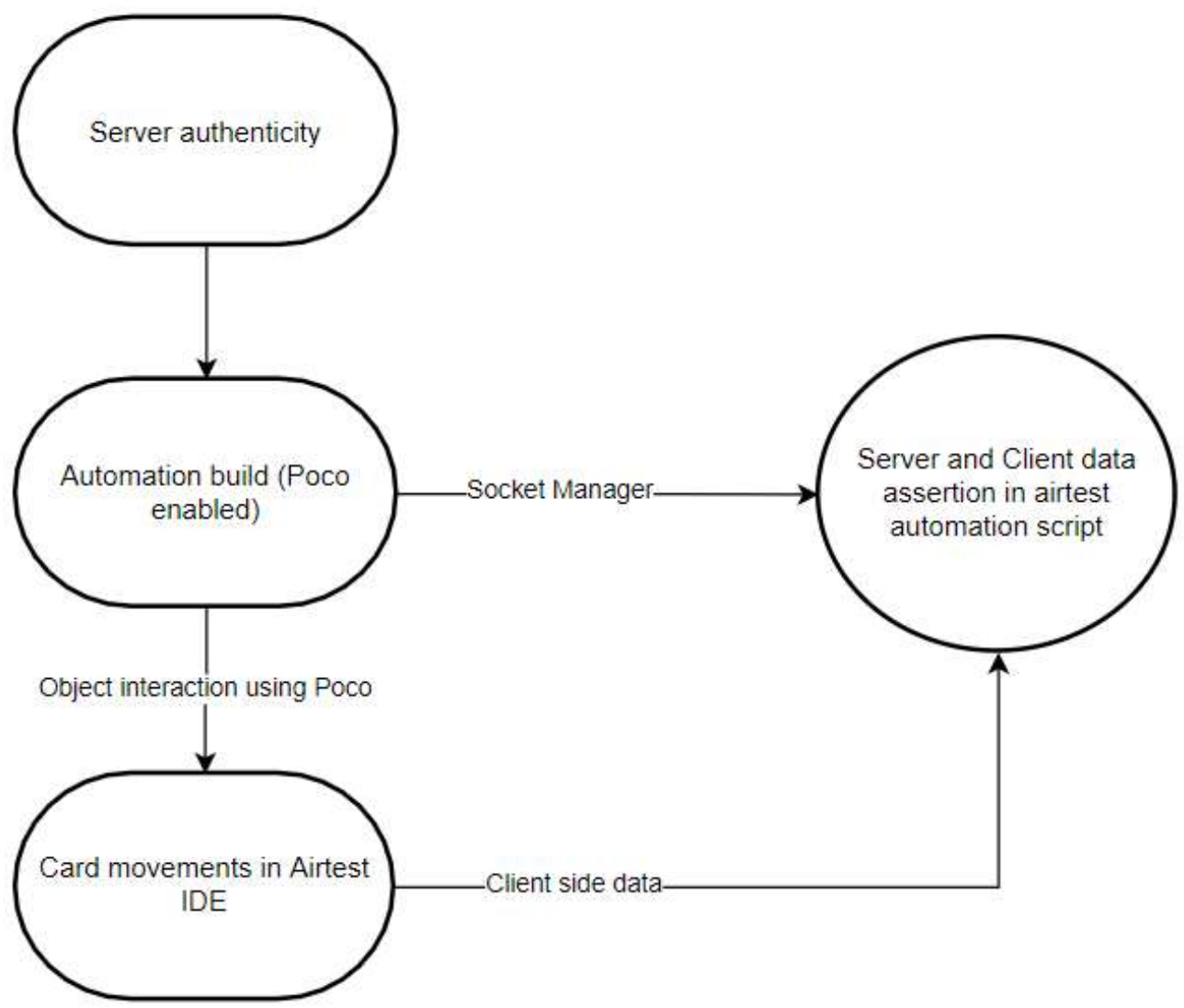

Fig. 3: Layers of communication 


\section{Remote Execution}

Airtest has a feature to run the test cases using CLI which has been used to move the test environment over a remote server which made it more accessible and executable at any time of the day. The ADB remote connection was used which is provided by Airtest that enables the use of any cloud emulators to run the test cases. We used the Genymotion[7] cloud solution to run multiple emulators on a different server. Through the CLI commands we can specify the specific test cases to run and the emulator over which we want the execution to happen.

Airtest provides a built-in report generator that automatically generates an HTML report of the entire execution process along with images and execution details. The report format can be customized. Using the CICD pipeline this whole process has been automated and for every execution the mail to the stakeholders gets auto-delivered.

\section{CONCLUSION}

We went ahead with the current approach of automation after doing groundwork on some of the famous automation tools like Appium[5] and AltUnity[6]. Airtest was the most viable option for us because of numerous points as shown in the comparison table below:

\begin{tabular}{|c|c|c|c|}
\hline Features & Airtest & Appium & AltUnity \\
\hline Unity support & Yes & No & Yes \\
\hline Multiplatform support & Yes & Yes & No \\
\hline Single codebase & Yes & Yes & NA \\
\hline Image recognition & Yes & No & No \\
\hline Multi-language support & No & Yes & No \\
\hline $\begin{array}{l}\text { Finding components uniquely } \\
\text { amongst multiple occurrences }\end{array}$ & Yes & No & Yes \\
\hline $\begin{array}{l}\text { Operable on a non-visible } \\
\text { frame }\end{array}$ & Yes & Yes & Yes \\
\hline $\begin{array}{l}\text { Independent executable } \\
\text { environment }\end{array}$ & Yes & No & No \\
\hline $\begin{array}{l}\text { Find objects outside the } \\
\text { viewport }\end{array}$ & No & No & Yes \\
\hline Inbuilt report generation & Yes & No & No \\
\hline Execution time & Moderate & Moderate & Comparatively faster \\
\hline
\end{tabular}


Since Airtest supports multiple environments and plugins for selenium within its IDE. This becomes useful when expanding a project to multiple platforms with minimal effort.

\section{REFERENCES}

1. Airtest [Online]. Available: https://Airtest.netease.com/

2. NetEase [Online]. Available: https://www.neteasegames.com/

3. Unity [Online]. Available: https://unity.com/

4. Poco [Online]. Available: https://github.com/AirtestProject/Poco

5. Appium [Online]. Available: https://appium.io/

6. AltUnity [Online]. Available: https://altom.gitlab.io/altunity/altunitytester/

7. Genymotion [Online]. Available: https://www.genymotion.com 